\title{
ELOVL2: a novel tumor suppressor attenuating tamoxifen resistance in breast cancer
}

\section{Dawoon Jeong}

Dongguk University - Seoul Campus: Dongguk University

\section{Juyeon Ham}

Dongguk University - Seoul Campus: Dongguk University

\section{Hyeon Woo Kim}

Dongguk University - Seoul Campus: Dongguk University

\section{Heejoo Kim}

Dongguk University - Seoul Campus: Dongguk University

\section{Hwee Won Ji}

Dongguk University - Seoul Campus: Dongguk University

\section{Sung Hwan Yun}

Dongguk University - Seoul Campus: Dongguk University Jae Eun Park

Dongguk University - Seoul Campus: Dongguk University

\section{Keun Seok Lee}

National Cancer Center

Heein Jo

National Cancer Center

Jai Hong Han

National Cancer Center

So-Youn Jung

National Cancer Center

\section{Seeyoun Lee}

National Cancer Center

\section{Eun Sook Lee}

National Cancer Center

Han-Sung Kang

National Cancer Center

Sun Jung Kim ( $\nabla$ sunjungk@dongguk.edu )

Dongguk University - Seoul Campus: Dongguk University https://orcid.org/0000-0001-6818-3956

\section{Research}


Keywords: breast cancer, epigenetics, methylation, tamoxifen resistance

Posted Date: September 8th, 2020

DOI: https://doi.org/10.21203/rs.3.rs-69357/v1

License: (c) (1) This work is licensed under a Creative Commons Attribution 4.0 International License. Read Full License 


\section{Abstract \\ Background}

To comprehensively understand the molecular mechanism of tamoxifen resistance (TamR) acquisition by epigenetically regulated genes, it is essential to identify pivotal genes by genome-wide methylation analysis and verify their function in xenograft animal model and cancer patients.

\section{Methods}

The MCF-7/TamR breast cancer cell line was developed and a genome-wide methylation array was performed. The methylation and expression of ELOVL2 was validated in cultured cells, xenografted tumor tissue, and breast cancer patients by methylation-specific PCR, qRT-PCR, Western blot analysis, and immunohistochemistry. Deregulation of ELOVL2 and THEM4 was achieved using siRNA or generating stable transfectants. Tam sensitivity, cell growth, and apoptosis were monitored by colorimetric and colony formation assay and flow cytometric analysis. Pathway analysis was performed to generate networks for the differentially methylated genes in the MCF-7/TamR cells and for the differentially expressed genes in the ELOVL2-overexpressing cells.

\section{Results}

Genome-wide methylation analysis in the MCF-7/TamR cells identified elongation of very-long chain fatty acid protein 2 (ELOVL2) to be significantly hypermethylated and downregulated, which was further verified in the tumor tissues from TamR breast cancer patients $(n=28)$ compared with those from Tamsensitive (TamS) patients $(n=33)(P<0.001)$. Immunohistochemical analysis of tissues from cancer patients showed lower expression of ELOVL2 in the TamR than TamS tissues. Growth of the MCF7/TamR cells overexpressing ELOVL2 was retarded in cell culture and also in xenograft tumor tissue. Strikingly, ELOVL2 attenuated resistance to Tam up to $70 \%$ judged by the colorimetric and colony formation assay and xenograft mouse model. ELOVL2 contributed to the recovery of Tam sensitivity by regulating a group of genes in the AKT and ERa signaling pathways, e.g., THEM4, which plays crucial roles in drug resistance.

\section{Conclusions}

ELOVL2 was hypermethylated and downregulated in TamR breast cancer patients compared with TamS patients. ELOVL2 is responsible for the recovery of Tam sensitivity. AKT- and ERa-hubbed networks are pivotal in ELOVL2 signaling, where THEM4 contributes to the relaying ELOVL2 signaling. This study implies that deregulation of a gene in fatty acid metabolism can lead to drug resistance, giving insight into the development of a new therapeutic strategy for drug-resistant breast cancer. 


\section{Background}

Tamoxifen is a non-steroidal antagonist of the estrogen receptor. It has been the first choice for adjuvant therapy in estrogen receptor-positive breast cancer as it reduces cancer recurrence and the annual mortality rate [1]. Despite the obvious benefits, $40 \%$ of breast cancer patient show cancer recurrence 510 years after initial therapy, which is one of the major setbacks for the clinicians [2]. This is partly because of the complexity of the signaling pathways that influence estrogen-mediated regulation in breast cancer $[3,4]$. Thus, identifying the key molecular markers and elucidating the molecular mechanism of drug resistance are pivotal for offering appropriate treatment options to cancer patients. During the course of tamoxifen-resistance (TamR) acquisition, cancer cells undergo cellular as well as molecular changes. A key change in these cells is increased proliferation and decreased apoptosis via BAX and BCL2 regulation [5]. In addition, the TamR cells show greater stemness phenotype by overexpressing Nanog, Oct3/4, and Sox2 [6].

Complex factors/pathways are attributed to TamR cells, including the activation of estrogen receptor (ER) signaling, up-regulation of growth factors (HER2, EGFR, FGFR, and IGF1R), alterations in RTK, a crosstalk among them, and consequently, the deregulation of the PI3K/AKT/mTOR pathway [7]. Cyclin D1/CDK4/6 complex is a target of the PI3K/AKT/mTOR pathway and has also been shown to crosstalk with the ER signaling pathway [8]. Previous studies have shown different Tam targets and their dysregulation from ER in TamR cancer, e.g., androgen receptor [9], Hedgehog signaling pathway [10], and non-coding RNAs [11], suggesting that the mechanism of TamR is far more complicated than just the modulation of ERassociated activity.

Therapeutic strategies for treating TamR cancer are under development, mainly targeting RTK pathways and PI3K/AKT/mTOR axis [12]. Further, cell cycle proteins can be targeted, for example, by using CDK4/ 6 inhibitors in combination with Tam [13]. Other approaches include targeting AKT pathway [14] and MYC [15] that are highly expressed in TamR cancer cells. However, clinical improvement has only been modest for these approaches till date; this may be because the affected pathways differ between patients. Thus, establishing accurate prognostic markers would hold the key to effective therapy for TamR breast cancer.

ELOVL2 is a member of the mammalian microsomal ELOVL fatty acid enzyme family, involved in the elongation of very long-chain fatty acids required for various cellular functions in mammals [16]. A study using Elovl2 ${ }^{-/-}$mice reaffirmed the importance of ELOVL2 for the elongation activity in rodents [17]. A prime characteristic of the gene is that the $\mathrm{CpG}$ near the gene exhibits consistent age-related changes in various tissues [18]. These strong associations have led to the development of a predictor that can accurately estimate the chronological age based on the methylation levels at the specific CpG site [19]. However, the relationship of ELOVL2 with cancer occurrence or development is unknown. Furthermore, no role of ELOVL2 in cancer drug resistance has been elucidated. In this study, ELOVL2 was identified to be downregulated by hypermethylation in TamR breast cancer. The involvement of ELOVL2 in the recovery of Tam sensitivity was suggested by presenting experimental evidence via in vitro as well as in vivo xenograft animal models. It was also suggested that ELOVL2 is a novel tumor suppressor by showing its 


\section{Materials And Methods}

\section{Cell culture and establishment of tamoxifen-resistant MCF- 7 (MCF-7/TamR) cells}

The human epithelial breast cancer cell line MCF-7 was obtained from the American Type Culture Collection (ATCC, Manassas, VA, USA) and was cultured under a humidified condition at $37^{\circ} \mathrm{C}, 5 \% \mathrm{CO}_{2}$ in RPMI 1640 medium (Gibco BRL, Carlsbad, CA, USA) containing 10\% fetal bovine serum (Capricorn, Germany) and $2 \%$ penicillin/streptomycin (Capricorn). All cells were used within 12 passages after resuscitation of stocks. The MCF-7/TamR cells were generated by culturing MCF-7 cells in the presence of 4-hydroxytamoxifen (Tam) (Sigma-Aldrich, St. Louis, MO, USA) in complete RPMI 1640 medium. The cells were continuously exposed to increasing concentrations of Tam up to $160 \mathrm{nM}$ over a period of 22 weeks, during which the medium was changed twice a week.

\section{Study subjects}

Solid tissues and slide-mounted formalin-fixed paraffin-embedded (FFPE) tissue sections of tumor samples were obtained from patients who underwent surgery between 2012 and 2013 at the National Cancer Center (NCC) in Korea. TamS tissues were obtained from patients who showed a clinical response to Tam, i.e., no tumor recurrence $(n=33)$. TamR tissues were obtained from patients who subsequently developed TamR (defined as disease recurrence while administering Tam; $n=28$ ). Clinical details are presented in Table S1. All patients provided written informed consent to donate the removed tissues to NCC in Korea, and samples were obtained according to the protocols approved by the Research Ethics Board of NCC.

\section{Generation of stable cell lines}

Lentiviral particles with control clones and human ELOVL2 ORF clones containing C-terminal mGFP tag were purchased from OriGene (Rockville, MD, USA). MCF-7 and MCF-7/TamR cells were seeded at a density of $5 \times 10^{3}$ cells/well in a 96-well plate 1 day before transduction. The next day, the cells were infected with lentivirus for $4 \mathrm{~h}$ in the presence of $8 \mu \mathrm{g} / \mathrm{mL}$ polybrene (Sigma-Aldrich), and then the medium was replaced with a fresh complete medium. After $72 \mathrm{~h}$, the cells were selected using $1 \mu \mathrm{g} / \mathrm{mL}$ puromycin (Thermo Fisher Scientific, Waltham, MA, USA) for 10 days.

\section{Cell Transfection}

siRNAs against ELOVL2 and THEM4 were purchased from Bioneer (Daejeon, Korea), and an ELOVL2overexpressing vector was developed using the pEZ-MT02 plasmid vector (GeneCopoeia, Rockville, MD, USA) by CosmoGenetech (Seoul, Korea). All siRNAs were diluted in Opti-MEM Medium (Gibco BRL) with Lipofectamine RNAiMAX (Invitrogen, Carlsbad, CA, USA), and the mixture was incubated for $5 \mathrm{~min}$. The 
cells were transiently transfected at final concentrations of $20 \mathrm{nM}$ or $40 \mathrm{nM}$ with siRNA following the manufacturer's instructions. Overexpression vectors $(2 \mu \mathrm{g})$ were transfected into the cells using Lipofectamine 3000 transfection reagent (Invitrogen). After $24 \mathrm{~h}$ of transfection, cells were harvested and used for the following experiments. All results for the optimization of transfection are demonstrated in Fig. S1.

\section{Cell proliferation assay}

The cell growth rate was monitored by colony formation assay and colorimetric assay using CCK-8 reagent (Dojindo, Kumamoto, Japan). In all, $3 \times 10^{3}$ cells/well were seeded onto a 96-well plate and cultured up to 5-7 days. Following staining with CCK-8 solution according to the provided instructions, optical densities were measured on a microplate reader (Sunrise, Tecan, Switzerland) and $\mathrm{OD}_{595}$ was eliminated from the $\mathrm{OD}_{450}$. For colony formation assay, cells were seeded at a density of $3 \times 10^{3}$ cells/dish on a $60-\mathrm{mm}$ culture dish. After transfection and Tam treatment, cells were maintained in a $5 \%$ $\mathrm{CO}_{2}$ incubator $\left(37^{\circ} \mathrm{C}\right)$ for $14-20$ days. Colonies were fixed with a 7:1 mixture of methanol and acetic acid, stained using $0.2 \%$ crystal violet (Gibco BRL), and counted with ImageJ software (NIH, MD, USA).

\section{Flow cytometric analysis}

Apoptosis was analyzed using an APC Annexin V Apoptosis Detection Kit with PI (BioLegend, San Diego, CA, USA). Annexin V staining was performed for cells diluted in Annexin V binding buffer for 8 min followed by propidium iodide $(\mathrm{PI})$ reagent treatment for $10 \mathrm{~min}$. Samples were measured using an Accuri C6 flow cytometer (BD Biosciences, San Jose, CA, USA) with 488-nm and 640-nm lasers. To monitor Tam uptake by cells, $1 \times 10^{6}$ cells seeded in a 60-mm dish were treated with FLTX1 (Aobious, Gloucester, MA, USA, AOB4054) for $2 \mathrm{~h}$ at final concentration of $10 \mu \mathrm{M}$. Cells were then harvested after washing with PBS, and a concentration of $1 \times 10^{6}$ cells $/ \mathrm{mL}$ was prepared. FLTX1 fluorescence was detected with Becton Dickinson FACSAria III (BD Biosciences) and analyzed with the Flowing Software 2.5 (http://flowingsoftware.btk.fi/).

\section{Tam sensitivity assay}

Alterations in sensitivity to Tam were measured by cytotoxicity assay. Briefly, $1 \times 10^{4}$ cells were seeded per well in a 96-well plate and transfected with recombinant cDNA-harboring plasmids and/or siRNAs. On the following day, Tam dissolved in sterile-filtered ethanol was added to cells at final concentrations of 0 , $0.05,0.1,0.5$, and $2 \mu \mathrm{M}$ with a final ethanol concentration $0.1 \%$. After $24 \mathrm{~h}, 10 \mu \mathrm{L}$ of CCK-8 solution was added to each well and the plate was incubated for $90 \mathrm{~min}$. Following this, the plate was read at $450 \mathrm{~nm}$ on a plate reader.

\section{Methylation and expression microarray experiment}

Genome-wide methylation analysis was performed with Macrogen (Seoul, Korea) on Illumina Infinium Human Methylation 450K and Illumina Infinium Methylation EPIC BeadChip (Illumina, San Diego, CA, USA) covering over 450,000 and $850,000 \mathrm{CpG}$ sites, respectively, to compare the DNA methylation profiles 
between MCF-7 and MCF-7/TamR. All arrays were processed with Illumina GenomeStudio v2011.1. To identify global gene expression profiles, total RNA of MCF-7/TamR/ORF NC or MCF-7/TamR/ELOVL2 ORF cells was profiled using the SurePrint G3 Human Gene Expression $8 \times 60 \mathrm{~K}$ v3 microarray technology (Agilent, Santa Clara, CA, USA) containing 58,201 probes by Lugen Sci (Seoul, Korea). Agilent Feature Extraction software (v11.0.1.1) was used to extract and process raw data. The microarray data are deposited in the GEO database website (http://www.ncbi.nlm.nih.gov/geo/) with the SuperSeries accession number GSE132617: expression array, GSE132614; methylation array, GSE132615 and GSE132616.

\section{Pathway and clustering analysis}

Ingenuity Pathway Analysis tool (Ingenuity Systems, Redwood City, CA, USA) was used to generate significant networks and biological functions for the differentially methylated genes within the promoter $(|\Delta \beta| \geq 0.2, P$-value $<0.05)$ by acquisition of Tam resistance, and for the differentially expressed genes (|fold changel $\geq 2, P$-value $<0.05$ ) by overexpressing ELOVL2 in MCF-7/TamR. Genes with significant changes were clustered using Clustering 3.0 software (http://bonsai.hgc.jp/ mdehoon/software/cluster/) and the results were visualized using the TreeView v1.1.6 program (http://jtreeview. sourceforge.net/).

\section{Methylation-specific PCR (MSP) and quantitative real-time RT-PCR (qPCR)}

MSP was performed to determine the methylation level of specific CpG sites, as previously described [20]. Briefly, DNA and RNA from FFPE sections were extracted using the RecoverAll Multi-Sample RNA/DNA Workflow (Invitrogen). Total DNA and RNA were prepared using ZR-Duet DNA/RNA MiniPrep kit (Zymo research, Irvine, CA, USA) from solid tissues and cultured cells. For preparing samples for methylation analysis, the genomic DNA was treated with bisulfite using a Zymo Research EZ DNA Methylation Kit (Zymo Research). Demethylation of the cytosine residues was achieved by exposing the cells to culture media containing a methyltransferase inhibitor, 5-Aza-2'-deoxycytidine (Aza) (Sigma-Aldrich), at a concentration of $5 \mu \mathrm{M}$ for $72 \mathrm{~h}$. PCR was conducted using 4-8 ng of DNA, and the yielded signals were calculated. To identify the transcript level of coding genes, cDNA was synthesized using a ReverTra Ace qPCR RT MasterMix with gDNA Remover kit (Toyobo, Osaka, Japan). qPCR analysis was conducted using KAPA SYBR FAST qPCR Kit (Kapa Biosystems, Wilmington, MA, USA) on an ABI 7300 instrument (Applied Biosystems, Foster City, CA, USA). Oligonucleotide primers were purchased from Bionics (Daejeon, Korea) (Table S2).

\section{Western blot analysis}

Protein extraction from cultured cells and Western blot analysis were performed as previously described [21]. The following antibodies were used: anti-ELOVL2 (1:500, Bioss, Woburn, MA, USA, bs-7053R), antiTHEM4 (1:500, Abcam, Cambridge, MA, USA, ab106435), anti- $\beta$-Actin (1:1000, Bioss, bs-0061R), antiphospho-AKT (1:300, Bioss, bs-5182R), anti-AKT (1:2500, Abcam, ab179463), and HRP-conjugated antirabbit IgG antibody (1:1000, GeneTex, Irvine, CA, USA, GTX213110-01). The bands on the membrane were 
detected using the ECL reagent (Abfrontier, Seoul, Korea) and analyzed with Image Lab software (Bio-Rad, Herculer, CA, USA). The whole blot can be accessed in Fig. S2.

\section{Tumor xenograft experiments}

All mouse experiments were approved by the Institutional Animal Care and Use Committee (IACUC) of Dongguk University (No: IACUC-2017-010-1). We used 6- to 7-week-old female BALB/c nude mice (Orient Bio, Seongnam, Korea) for MCF-7 and MCF-7/TamR-derived xenograft models. The mice were anesthetized with a mixture of isoflurane (Piramal Critical Care, Mettawa, IL, USA) and oxygen, and administered $17 \beta$-estradiol pellets ( $0.72 \mathrm{mg} /$ pellet total dose; Innovative Research of America, Sonnasota, FL, USA) subcutaneously in the lateral neck area. On the next day, subcutaneous injections of $1 \times 10^{7}$ breast cancer cells resuspended with $100 \mu \mathrm{L}$ of 1:1 mixture of PBS (Gibco BRL) and Matrigel (BD Biosciences, Bedford, MA, USA) were administered to the mice. Tumor growth was monitored weekly, and tumor volumes were calculated based on the following formula: length $\times$ width $^{2} \times 0.5$. When tumor sizes reached approximately $100 \mathrm{~mm}^{3}$, the mice were randomized into two groups for Tam treatment (SigmaAldrich). One group received intraperitoneal administration of $100 \mu \mathrm{L}$ of $1 \mathrm{mg} / \mathrm{kg}$ Tam in corn oil (SigmaAldrich) and the other group was injected with a vehicle control for 5 days a week during the experiment. After 7 weeks of implantation, animals were sacrificed and tumors were harvested. The cancer tissues were fixed in $4 \%$ paraformaldehyde and embedded in paraffin blocks for histological analysis by Logone Bio (Seoul, Korea).

\section{Immunohistochemical staining}

Immunohistochemical analysis was performed using tumor tissues of xenograft mice. To do this, paraffin blocks were sectioned $10 \mu \mathrm{m}$ thick, organized into slides, and rehydrated through a graded ethanol series. Endogenous peroxidase activity in sections was ceased with $0.3 \% \mathrm{H}_{2} \mathrm{O}_{2}$ treatment for 15 min and then rabbit anti-ELOVL2 (1:400, Bioss, bs-7053R) or rabbit anti-THEM4 (1:100, Abcam, ab106435) was applied for $1 \mathrm{~h}$ at room temperature followed by incubation with horseradish peroxidaseconjugated anti-rabbit antibodies (Dako, Glostrup, Denmark, K4003). Liquid diaminobenzidine tetrahydrochloride (DAB) (Dako, K3468) was used as a chromogen to detect horseradish peroxidase activity. After counterstaining with Mayer's hematoxylin, immunohistochemical images were generated using panoramic MIDI scanner (3Dhistech, Budapest, Hungary). The ImageJ program (NIH) was used to profile the DAB-positive areas of immunohistochemical images.

\section{Statistical analysis}

For microarray data, observations with adjusted $P$-values $\geq 0.05$ were removed and were excluded from further analysis. Adjustments were made to control for false discoveries. Following adjustments, the remaining genes were defined as differentially methylated if they displayed an increased or decreased methylation level which was equal to or higher than 0.2 compared with the control, or differentially expressed if they displayed at least a 2-fold difference compared with the control. Student's t-test was implemented to demonstrate statistical significance for all data from qPCR, MSP, IHC, and Western blot analysis comparing samples and control groups. Chi-squared test was used to analyze the differences in 
the rate of each variable for tumor tissues. Statistical analyses were conducted using SPSS for Windows, release 17.0 (SPSS Inc., Chicago, IL, USA). The results are expressed as the mean \pm standard error and considered statistically significant at $P$-value $<0.05$.

\section{Results}

\section{Generation of MCF/TamR cells}

As a prerequisite to explore the molecular mechanism of TamR, a MCF-7/TamR cell line was established by exposing the MCF-7 cells to increasing concentrations of Tam up to $160 \mathrm{nM}$ for 22 weeks (Fig. S3a). The finally developed MCF-7/TamR cells showed a higher growth rate compared with their parental cells (Fig. S3b) and also a large number of cells survived against high concentrations of Tam ( 0.1 and 0.5 ump) as judged by the colony formation assay (Fig. S3c). The apoptosis rate was lower in TamR cells and less affected by Tam than in the MCF-7 cells (Fig. S3d and Fig. S4), possibly explaining the higher growth and survival rate in the presence of Tam. The acquisition of TamR was further monitored by examining a few marker genes, the expressions of which were previously known to be altered in the course of TamR acquisition. Thus, EGFR, CCND1, CD146, and BCAR3 were upregulated, whereas BAG1 and IGF1 were downregulated, as previously observed [22-25], confirming appropriate generation of MCF-7/TamR cells (Fig. S3e). Notably, the level of Tam inside the TamR cells was not decreased compared with that in the parental MCF-7 cells after the two cell types were equally treated with Tam, indicating that the resistance is not caused by a net decrease of the drug transport across the plasma membrane (Fig. S3f)

\section{ELOVL2 is downregulated by DNA hypermethylation in TamR breast cancer}

Genome-wide methylation analysis was performed in duplicates for each sample of MCF-7 and MCF$7 /$ TamR cells. Comparison of the two cell types showed hyper- and hypo-methylation with $|\Delta \beta| \geq 0.2$ at 331 and $94 \mathrm{CpG}$ sites, respectively, corresponding to 356 unique genes (Fig. 1a). Among highly altered genes, $\operatorname{ESR} 1(\Delta \beta=0.35), \operatorname{MAGED1}(\Delta \beta=0.3)$, and $\operatorname{RASAL} 1(\Delta \beta=0.36)$ were listed, alteration in methylation of which in TamR cells has been previously known [26-28], indicating the reliability of MCF-7/TamR cells developed in the current study. The microarray data were also verified by examining the expression of five randomly selected genes from the highly altered genes via qPCR. Consequently, hypermethylated SCL19A1, SKAP1, and ELOVL2 were downregulated, whereas hypomethylated CD59 and MMP1 were upregulated (Fig. 1b), supporting the close relationship between methylation and expression. Next, the 356 genes were examined for functional inter-relatedness using the IPA software tool. The top network with the highest confidence was "Skeletal and Muscular Disorders, Cellular Assembly and Organization, Connective Tissue Development and Function" (Fig. 1C). Canonical pathway analysis identified "Neuroactive ligand-receptor interaction" as the predominant pathway (Fig. 1d). Disease and function annotation analysis indicated that genes associated with "Cancer" and "Cell death and survival" are frequently included (Fig. 1e). 
To identify a novel and pivotal marker that contributes to the acquisition of TamR, ELOVL2 in Table S3 was selected because the gene showed significant hypermethylation $(\Delta \beta=0.49)$, but its role in cancer or drug resistance is not yet known. Both transcripts and proteins were downregulated in the MCF-7/TamR cells compared with those in MCF-7 cells (Fig. 1b and 2a). Induction of lower methylation by treating the MCF-7/TamR cells with Aza, a methyltransferase inhibitor, upregulated ELOVL2, suggesting an epigenetic regulation of the gene (Fig. 2b). Next, the methylation and expression of the gene were examined in breast cancer tissues obtained from patients showing resistance to clinical treatment with Tam. The result indicated that ELOVL2 was hypermethylated and downregulated in the TamR cancer tissues $(n=$ 28) compared with the Tamoxifen sensitive (TamS) tissues $(n=33)(P<0.001)$ (Fig. 2c).

Immunohistochemical analysis of tissues from cancer patients showed lower expression of ELOVL2 in the TamR tissues than in the TamS tissues (Fig. 2d). The rate of distant metastasis-free survival (DMFS) for breast cancer patients, which was investigated through the GOBO database, indicates that lower rates of DMFS were observed in cancer patients with lower expression of ELOVL2, whereas higher rates of DMFS were observed in patients with a higher ELOVL2 expression $(P<0.05)$ (Fig. 2e).

\section{ELOVL2 inhibits MCF-7/TamR cell proliferation and recovers Tam sensitivity}

To obtain information about the role of ELOVL2 in the acquisition of TamR, its effect on cell growth and drug sensitivity recovery was examined after constructing ELOVL2-overexpressing MCF-7/TamR cells (MCF-7/TamR-ELOVL2 ORF). The growth rate of MCF-7/TamR-ELOVL2 ORF cells was retarded up to $18 \%$ compared with that of MCF-7/TamR cells (Fig. 3a). In the presence of Tam, the growth rate of the MCF7/TamR-ELOVL2 ORF cells was further retarded, especially at Tam concentrations of $<0.5 \mu \mathrm{M}$ (Fig. 3b). This observation was also reproduced in the cell survival experiment of colony formation assay, where a lower number of colonies were observed in the MCF-7/TamR-ELOVL2 ORF cells under Tam pressure (Fig. 3c).

Next, the effect of ELOVL2 on the recovery of Tam sensitivity was monitored in a mouse xenograft model where the cancer cells were subcutaneously injected. As a result, MCF-7/TamR cells grew faster than MCF-7 (Fig. 4a) and showed larger tumor volume in all the eight mice sacrificed 7 weeks after injection (Fig. 4b). A decrease in ELOVL2 protein expression in the tumor was observed by Western blot and immunohistochemical analysis (Fig. $4 \mathrm{c}$ and d). The animals implanted with MCF-7/TamR-ELOVL2 ORF showed retarded tumor growth compared with animals with MCF-7/TamR cells (Fig. 4e and f), indicating the tumor suppressive activity of ELOVL2. Furthermore, the tumors over-expressing ELOVL2 showed an increased sensitivity to Tam by representing a smaller tumor size (Fig. 4e and f).

\section{ELOVL2 resists Tam by suppressing the AKT pathway}

To get an insight into the regulatory mechanism of ELOVL2 for drug resistance, a genome-wide expression assay was performed in duplicates for identifying target genes. A comparison of the expression profile between MCF-7/TamR and MCF-7/TamR- ELOVL2 ORF revealed 969 genes that were significantly altered (expression level change $>2$ ) (Fig. 5a). IPA analysis identified "Cardiovascular 
Disease, Cell-To-Cell Signaling and Interaction, Inflammatory Response" pathway and "Behavior, Reproductive System Development and Function, Cardiac Infarction" pathway as the top first and second networks, respectively (Fig. 5b). In accordance with this, the canonical pathway analysis (Fig. 5c) and the disease and function analysis (Fig. 5d) predicted the immune-related pathway and cancer as the top categories, respectively. Notably, AKT and ERa are placed at the center of each network, interacting directly or indirectly with many genes in the pathways, such as THEM4, BMF, and FTO in the case of AKT pathway and HYOU1, CDC42EP2, and S100A9 in the case of ERa. The expression of the genes in the two pathways were further examined by qPCR and the result confirmed the same direction of expression alteration as observed in the expression array (Fig. S5). The AKT pathway is a key pathway responsible for cell metabolism, growth and division, apoptosis suppression, and angiogenesis [29]. In particular, THEM4 is known to promote AKT phosphorylation and functions as an oncogenic molecule in breast cancer [30].

\section{THEM4 is downregulated by ELOVL2 recovering Tam sensitivity}

To elucidate the molecular mechanism of how THEM4 induced TamR in association with ELOVL2, its expression was examined in cell lines and tumor tissues from xenografted mice. THEM4 was upregulated in the MCF-7/TamR cells compared with MCF-7 cells at both RNA and protein levels (Fig. 6a).

Overexpression of ELOVL2 in the MCF-7/TamR cells set back the THEM4 expression to a lower level than that observed in the control MCF-7/TamR cells (Fig. 6b). Western blot analysis (Fig. 6c) and immunohistochemical analysis (Fig. 6d, e) also found a similar expression profile for THEM4 in the tumor tissues of the xenografted mice, which were generated from MCF-7/TamR and MCF-7/TamR-ELOVL2 ORF. Subsequently, the association of THEM4 with ELOVL2 was tested with regard to TamR, cell growth, and apoptosis. siRNA-induced downregulation of THEM4 contributed to the recovery of Tam sensitivity as shown by the colony formation assay (Fig. 6f), and this effect was strengthened by ELOVL2 (Fig. 6g). The dye-based CCK assay using the same THEM4-siRNA and ELOVL2-overexpression strategy confirmed the results of the colony formation assay (Fig. 6h, i), suggesting an inhibitory and a stimulatory effect of ELOVL2 and THEM4, respectively, for the acquisition of TamR. However, the total apoptosis rate was not changed significantly, even though early and late apoptosis was decreased and increased, respectively, by the downregulation of THEM4 (Fig. S6).

Considering all the experimental findings from this study and the previous literature, which indicate a close association of AKT and THEM4 in the signaling pathway, it is suggested that ELOVL2 contributes to the recovery of TamR by regulating pivotal genes such as THEM4 in the AKT pathway (Fig. 7).

\section{Discussion}

This study aimed at identifying epigenetically regulated marker genes responsible for TamR in breast cancer patients and then elucidating the molecular mechanism to give an insight for the prevention and treatment of TamR recurrence in cancer patients. Occasionally, drug-resistant cancer cells have shown to 
actively pump the drug out of the cells, reducing the net amount of drug inside cells. This fact could partly explain drug resistance. For instance, a 10-fold lower Tam concentration was found in extracts from TamR tumors than that in TamS tumors in mice [31]. However, it is controversial whether all TamR cells acquire the high efflux rate, because the P-glycoprotein, an efflux pump that is known to bind to Tam, is not expressed in these tumors. Furthermore, the TamR cells generated and used in our study showed no significant change in Tam transport compared with the parental MCF-7 cells. Therefore, we speculated that genetic changes may have more strongly contributed to TamR than alterations in the drug efflux rate.

ELOVL2 has consistently shown hypermethylation and downregulation in TamR cancer, indicating its potential application as an epigenetic marker for the diagnosis of TamR cancer. However, no significant difference of methylation or expression between normal and cancer tissues of breast was found. In normal tissue, ELOVL2 has been known to undergo hypermethylation on the promoter DNA as one ages. The downregulation of ELOVL2 induced by the hypermethylation is not considered enough to drive cells into cancer, although suppression of ELOVL2 stimulated cell proliferation in our study. This may imply that the deregulation of ELOVL2 is crucial during the acquisition of TamR, rather than during the development of the primary cancer. Moreover, an association between ELOVL2 DNA methylation and future breast and colorectal cancer development has been observed [32].

ELOVL2 Expression has been previously known to be enhanced by ERa in breast cancer cells [33]. In this study, Tam exposure specifically abolished ELOVL2 expression. Our microarray analysis also revealed upregulation of ERa by ELOVL2, suggesting a positive feedback mechanism for the regulation of the two genes. In addition, many ERa-regulated genes such as HYOU1 (3.8-fold decrease), S100A9 (1.2-fold decrease), and CDC42EP2 (2.4-fold decrease) were also deregulated by ELOVL2. HYOU1 is a hypoxiainduced protein and its upregulation suppresses programmed cell death, contributing to invasiveness in breast cancer [34]. S100A9 has been identified to be expressed by epithelial cells involved in malignancy and its expression levels are inversely correlated with ERa in breast cancer [35]. CDC42EP2 is a member of the binder of Rho GTPases (Borg) family and little is known about its role in the disease [36].

The AKT pathway is a pivotal one wherein a few TamR-related genes have been identified [23]. In accordance, previously identified AKT-regulated genes also appeared in the ELOVL2-overexpression network, such as THEM4, BMF, and FTO (Fig. 5b). Furthermore, other genes involved in the AKT pathway, including mTOR, PIK3CA, and CREB1, were shown to be downregulated by ELOVL2 (Fig. S7a). Notably, the expression of Akt as well as the phosphorylated form was downregulated in a similar ratio by ELOVL2 (Fig. S7b). Therefore, the decrease of the phosphorylated form is caused by the lowered total AKT levels, suggesting that ELOVL2 regulates AKT and not p-AKT. The two networks closely communicating each other by sharing a few common genes. For example, NFAT 5 is regulated by an estrogen-induced microRNA [37] and the regulation is mediated via PI3K/AKT-signaling pathways [38]. In addition, A100A9, a calcium-binding protein that is highly expressed in malignant breast cancer, induces a decrease of ERa in MCF-7 cell [35], and inhibits PI3K/AKT pathway in pancreatic adenocarcinoma cells [39]. Collectively, the current study proposes that ELOVL2 is an integral signaling molecule of the AKT axis in ER-positive breast cancer cells. 
Further it is noteworthy that a representative aging marker gene is associated with drug resistance. ELOVL2 has shown to increase methylation at the promoter CpGs with aging, which accompanies a decreased expression with aging [18]. Considering the increased drug resistance and lower expression of ELOVL2 in cancer cells of patients, epigenetic aging could make cancer patients more vulnerable to acquisition of drug resistance. This gives us an insight on how to design the strategy for treating drugresistant cancers. Meanwhile, it should be mentioned that ELOVL2 function is not limited to epigenetic aging and drug resistance. The gene showed tumor suppressor-like activity by inhibiting cancer cell growth in cultures cancer cells as well as in the xenografted mouse model. Therefore, ELOVL2 is considered to have a wide spectrum of biological functions in addition to the fatty acid elongation activity.

Genome-wide methylation analysis has found that numerous genes were deregulated in addition to ELOVL2 in TamR cells, suggesting distortion of multiple pathways during the course of drug resistance acquisition. A genome-wide expression array also found $>1,200$ genes clustered into ERa function, cell cycle regulation, transcription/translation, and mitochondrial dysfunction [40]. Therefore, to completely understand the molecular mechanisms and to conquer TamR in cancer, a further comprehensive approach is needed.

\section{Conclusions}

Altogether, a genome-wide profile of epigenetic changes during TamR acquisition in breast cancer cells was constructed. ELOVL2 was identified as a marker that was hypermethylated and downregulated in TamR cancer compared with TamS cancer. ELOVL2 is responsible for the recovery of TamS, which was shown in an in vivo animal xenograft model. AKT- and ERa-hubbed networks are pivotal in ELOVL2 signaling, where THEM4 contributes to the relaying ELOVL2 signaling. This study is the first to identify a linkage between drug resistance and a gene involved in fatty acid synthesis. Our data may give credence to elucidating the mechanism of TamR cancer and to developing its treatment strategy.

\section{Abbreviations}

ELOVL2: elongation of very-long chain fatty acid protein 2; qPCR:quantitative real-time reverse transcription-polymerase chain reaction; MSP:methylation-specific PCR; IHC:immunohistochemistry; CCK8:cell counting kit-8; IPA:Ingenuity Pathway Analysis; Tam:4-hydroxytamoxifen; TamR:tamoxifen resistance; TamS:tamoxifen sensitivity; ER:estrogen receptor; THEM4:thioesterase superfamily member 4; AKT:protein kinase B; EGFR:epidermal growth factor receptor; IGF1:insulin-like growth factor 1; MMP1:matrix metalloproteinase-1; FFPE:formalin-fixed paraffin-embedded; ORF:open reading frame; Aza:5-Aza-2'-deoxycytidine; gDNA:genomic DNA; cDNA:complementary DNA; siRNA:small interfering RNA; PBS:phosphate buffered saline; FC:fold change; PI:propidium iodide; DMFS:distant metastasis-free survival; GEO:gene expression omnibus; GOBO:gene expression-based outcome

\section{Declarations}




\section{Availability of data and materials}

The data supporting the conclusions of this article are presented within the article and its additional files.

\section{Ethics approval and consent to participate}

All patients provided written informed consent to donate the removed tissues to NCC in Korea, and samples were obtained according to the protocols approved by the Research Ethics Board of NCC. All mouse experiments were approved by the Institutional Animal Care and Use Committee (IACUC) of Dongguk University (No: IACUC-2017-010-1).

\section{Consent for publication}

Written informed consent was obtained from all patients.

\section{Competing interest}

The authors declare no competing interest.

\section{Funding}

This work was supported by the Basic Science Research Program (NRF-2016R1D1A1B01009235) of the National Research Foundation of Korea funded by the Ministry of Education, Science and Technology. Dr H.S. Kang was supported by a grant provided by the National Cancer Center, Korea.

\section{Authors' contributions}

D.J. performed the majority of the experiments. J.H., H.W.K., and S.H.Y. contributed to biochemical experiments. H.K. and H.W.J. performed microarray analysis. E.S.L., S.L., S.Y.J., J.H.H., H.J., and K.S.L. performed surgeries and contributed to histology and immunohistochemistry. H.S.K. and S.J.K. designed the study, analyzed data, coordinated the experimental work, and wrote the manuscript with contribution from all authors.

\section{Acknowledgements}

The authors thank Ms. Seungyeon Lee and Ms. Hyunkyung Lee for their technical assistance.

\section{References}

1. Fisher B, Costantino JP, Wickerham DL, Cecchini RS, Cronin WM, Robidoux A, Bevers TB, Kavanah MT, Atkins JN, Margolese RG, et al. Tamoxifen for the prevention of breast cancer: current status of 
the National Surgical Adjuvant Breast and Bowel Project P-1 study. J Natl Cancer Inst. 2005;97(22):1652-62.

2. Gradishar WJ. Tamoxifen-what next? Oncologist 2004, 9(4):378-384.

3. Gao A, Sun T, Ma G, Cao J, Hu Q, Chen L, Wang Y, Wang Q, Sun J, Wu R, et al. LEM4 confers tamoxifen resistance to breast cancer cells by activating cyclin D-CDK4/6-Rb and ERalpha pathway. Nat Commun. 2018;9(1):4180.

4. Shah N, Jin K, Cruz LA, Park S, Sadik H, Cho S, Goswami CP, Nakshatri H, Gupta R, Chang HY, et al. HOXB13 mediates tamoxifen resistance and invasiveness in human breast cancer by suppressing ERalpha and inducing IL-6 expression. Cancer Res. 2013;73(17):5449-58.

5. Mirzapur P, Khazaei MR, Moradi MT, Khazaei M. Apoptosis induction in human breast cancer cell lines by synergic effect of raloxifene and resveratrol through increasing proapoptotic genes. Life Sci. 2018;205:45-53.

6. Arif K, Hussain I, Rea C, El-Sheemy M. The role of Nanog expression in tamoxifen-resistant breast cancer cells. Onco Targets Ther. 2015;8:1327-34.

7. Zhu Y, Liu Y, Zhang C, Chu J, Wu Y, Li Y, Liu J, Li Q, Li S, Shi Q, et al. Tamoxifen-resistant breast cancer cells are resistant to DNA-damaging chemotherapy because of upregulated BARD1 and BRCA1. Nat Commun. 2018;9(1):1595.

8. Vora SR, Juric D, Kim N, Mino-Kenudson M, Huynh T, Costa C, Lockerman EL, Pollack SF, Liu M, Li X, et al. CDK 4/6 inhibitors sensitize PIK3CA mutant breast cancer to PI3K inhibitors. Cancer Cell. 2014;26(1):136-49.

9. Ciupek A, Rechoum Y, Gu G, Gelsomino L, Beyer AR, Brusco L, Covington KR, Tsimelzon A, Fuqua SA. Androgen receptor promotes tamoxifen agonist activity by activation of EGFR in ERalpha-positive breast cancer. Breast Cancer Res Treat. 2015;154(2):225-37.

10. Ramaswamy B, Lu Y, Teng KY, Nuovo G, Li X, Shapiro CL, Majumder S. Hedgehog signaling is a novel therapeutic target in tamoxifen-resistant breast cancer aberrantly activated by PI3K/AKT pathway. Cancer Res. 2012;72(19):5048-59.

11. Miller TE, Ghoshal K, Ramaswamy B, Roy S, Datta J, Shapiro CL, Jacob S, Majumder S. MicroRNA221/222 confers tamoxifen resistance in breast cancer by targeting p27Kip1. J Biol Chem. 2008;283(44):29897-903.

12. Ali S, Rasool M, Chaoudhry H, Jha PNP, Hafiz P, Mahfooz A, Abdus Sami M, Azhar Kamal G, Bashir M. $S$ et al: Molecular mechanisms and mode of tamoxifen resistance in breast cancer. Bioinformation. 2016;12(3):135-9.

13. Rondon-Lagos M, Villegas VE, Rangel N, Sanchez MC, Zaphiropoulos PG. Tamoxifen Resistance: Emerging Molecular Targets. Int J Mol Sci 2016, 17(8).

14. Jin K, Park S, Teo WW, Korangath P, Cho SS, Yoshida T, Gyorffy B, Goswami CP, Nakshatri H, Cruz LA, et al. HOXB7 Is an ERalpha Cofactor in the Activation of HER2 and Multiple ER Target Genes Leading to Endocrine Resistance. Cancer Discov. 2015;5(9):944-59. 
15. Thomas S, Thurn KT, Raha P, Chen S, Munster PN. Efficacy of histone deacetylase and estrogen receptor inhibition in breast cancer cells due to concerted down regulation of Akt. PLoS One. 2013;8(7):e68973.

16. Gregory MK, Gibson RA, Cook-Johnson RJ, Cleland LG, James MJ. Elongase reactions as control points in long-chain polyunsaturated fatty acid synthesis. PLoS One. 2011;6(12):e29662.

17. Pauter AM, Olsson P, Asadi A, Herslof B, Csikasz RI, Zadravec D, Jacobsson A. Elovl2 ablation demonstrates that systemic DHA is endogenously produced and is essential for lipid homeostasis in mice. J Lipid Res. 2014;55(4):718-28.

18. Fernandez AF, Bayon GF, Urdinguio RG, Torano EG, Garcia MG, Carella A, Petrus-Reurer S, Ferrero C, Martinez-Camblor P, Cubillo I, et al. H3K4me1 marks DNA regions hypomethylated during aging in human stem and differentiated cells. Genome Res. 2015;25(1):27-40.

19. Hannum G, Guinney J, Zhao L, Zhang L, Hughes G, Sadda S, Klotzle B, Bibikova M, Fan JB, Gao Y, et al. Genome-wide methylation profiles reveal quantitative views of human aging rates. Mol Cell. 2013;49(2):359-67.

20. Kim SJ, Kelly WK, Fu A, Haines K, Hoffman A, Zheng T, Zhu Y. Genome-wide methylation analysis identifies involvement of TNF-alpha mediated cancer pathways in prostate cancer. Cancer Lett. 2011;302(1):47-53.

21. Lee S, Lee H, Jeong D, Ham J, Park S, Choi EH, Kim SJ. Cold atmospheric plasma restores tamoxifen sensitivity in resistant MCF-7 breast cancer cell. Free Radic Biol Med. 2017;110:280-90.

22. Viedma-Rodriguez R, Baiza-Gutman L, Salamanca-Gomez F, Diaz-Zaragoza M, Martinez-Hernandez G, Ruiz Esparza-Garrido R, Velazquez-Flores MA, Arenas-Aranda D. Mechanisms associated with resistance to tamoxifen in estrogen receptor-positive breast cancer (review). Oncol Rep. 2014;32(1):3-15.

23. Liang YK, Zeng, Xiao YS, Wu Y, Ouyang YX, Chen M, Li YC, Lin HY, Wei XL, Zhang YQ, et al. MCAM/CD146 promotes tamoxifen resistance in breast cancer cells through induction of epithelialmesenchymal transition, decreased ERalpha expression and AKT activation. Cancer Lett. 2017;386:65-76.

24. Lu S, Du Y, Cui F, Feng X, Ma Y, Liu H. Downregulation of BAG1 in T47D cells promotes resistance to tamoxifen via activation of the PI3K/Akt/mTOR signaling pathway. Oncol Rep. 2019;41(3):1901-10.

25. Chong K, Subramanian A, Sharma A, Mokbel K. Measuring IGF-1, ER-alpha and EGFR expression can predict tamoxifen-resistance in ER-positive breast cancer. Anticancer Res. 2011;31(1):23-32.

26. Selmin OI, Donovan MG, Skovan B, Paine-Murieta GD, Romagnolo DF. Arsenicinduced BRCA1 CpG promoter methylation is associated with the downregulation of ERalpha and resistance to tamoxifen in MCF7 breast cancer cells and mouse mammary tumor xenografts. Int J Oncol. 2019;54(3):86978.

27. Williams KE, Anderton DL, Lee MP, Pentecost BT, Arcaro KF. High-density array analysis of DNA methylation in Tamoxifen-resistant breast cancer cell lines. Epigenetics. 2014;9(2):297-307. 
28. Stone A, Valdes-Mora F, Gee JM, Farrow L, McClelland RA, Fiegl H, Dutkowski C, McCloy RA, Sutherland RL, Musgrove EA, et al. Tamoxifen-induced epigenetic silencing of oestrogen-regulated genes in anti-hormone resistant breast cancer. PLoS One. 2012;7(7):e40466.

29. Nitulescu GM, Van De Venter M, Nitulescu G, Ungurianu A, Juzenas P, Peng Q, Olaru OT, Gradinaru D, Tsatsakis A, Tsoukalas D, et al. The Akt pathway in oncology therapy and beyond (Review). Int J Oncol. 2018;53(6):2319-31.

30. Liu YP, Liao WC, Ger LP, Chen JC, Hsu TI, Lee YC, Chang HT, Chen YC, Jan YH, Lee KH, et al. Carboxylterminal modulator protein positively regulates Akt phosphorylation and acts as an oncogenic driver in breast cancer. Cancer Res. 2013;73(20):6194-205.

31. Osborne CK, Fuqua SA. Mechanisms of tamoxifen resistance. Breast Cancer Res Treat. $1994 ; 32(1): 49-55$.

32. Durso DF, Bacalini MG, Sala C, Pirazzini C, Marasco E, Bonafe M, do Valle IF, Gentilini D, Castellani G, Faria AMC, et al. Acceleration of leukocytes' epigenetic age as an early tumor and sex-specific marker of breast and colorectal cancer. Oncotarget. 2017;8(14):23237-45.

33. Gonzalez-Bengtsson A, Asadi A, Gao H, Dahlman-Wright K, Jacobsson A. Estrogen Enhances the Expression of the Polyunsaturated Fatty Acid Elongase Elovl2 via ERalpha in Breast Cancer Cells. PLoS One. 2016;11(10):e0164241.

34. Tsukamoto Y, Kuwabara K, Hirota S, Kawano K, Yoshikawa K, Ozawa K, Kobayashi T, Yanagi H, Stern DM, Tohyama M, et al: Expression of the 150-kd oxygen-regulated protein in human breast cancer. Lab Invest 1998, 78(6):699-706.

35. Bao YI, Wang A, Mo J. S100A8/A9 is associated with estrogen receptor loss in breast cancer. Oncol Lett. 2016;11(3):1936-42.

36. Farrugia AJ, Calvo F. The Borg family of Cdc42 effector proteins Cdc42EP1-5. Biochem Soc Trans. 2016;44(6):1709-16.

37. Zierau O, Helle J, Schadyew S, Morgenroth Y, Bentler M, Hennig A, Chittur S, Tenniswood M, Kretzschmar G. Role of miR-203 in estrogen receptor-mediated signaling in the rat uterus and endometrial carcinoma. J Cell Biochem. 2018;119(7):5359-72.

38. Martin VA, Wang WH, Lipchik AM, Parker LL, He Y, Zhang S, Zhang ZY, Geahlen RL. Akt2 inhibits the activation of NFAT in lymphocytes by modulating calcium release from intracellular stores. Cell Signal. 2012;24(5):1064-73.

39. Moz S, Basso D, Bozzato D, Galozzi P, Navaglia F, Negm OH, Arrigoni G, Zambon CF, Padoan A, Tighe $P$, et al. SMAD4 loss enables EGF, TGFbeta1 and S100A8/A9 induced activation of critical pathways to invasion in human pancreatic adenocarcinoma cells. Oncotarget. 2016;7(43):69927-44.

40. Huber-Keener KJ, Liu X, Wang Z, Wang Y, Freeman W, Wu S, Planas-Silva MD, Ren X, Cheng Y, Zhang $Y$, et al. Differential gene expression in tamoxifen-resistant breast cancer cells revealed by a new analytical model of RNA-Seq data. PLoS One. 2012;7(7):e41333.

\section{Figures}


a

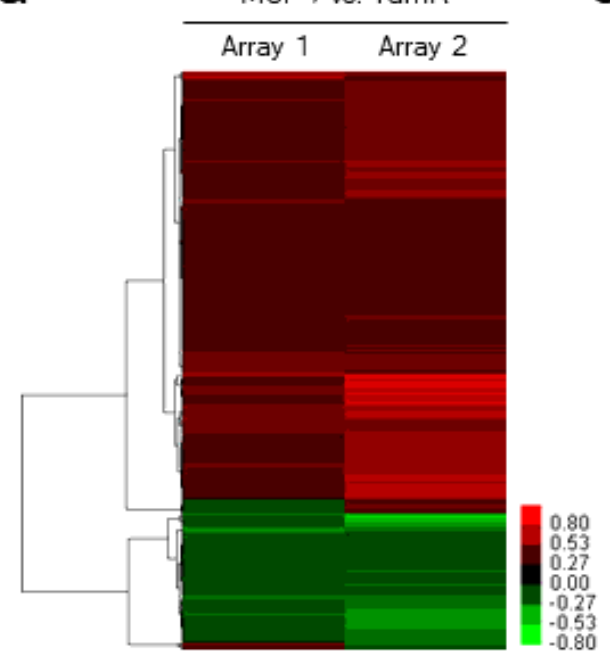

b

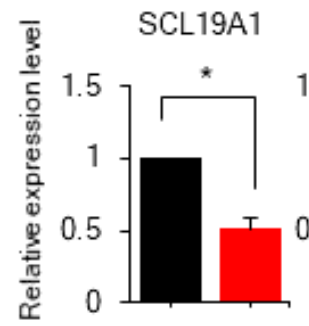

SKAP1
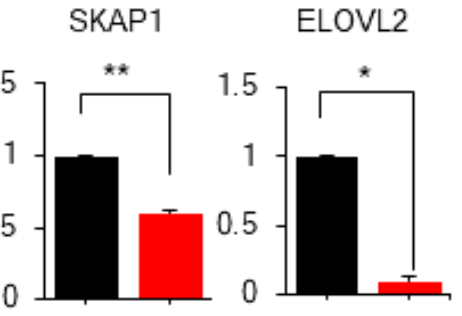

d

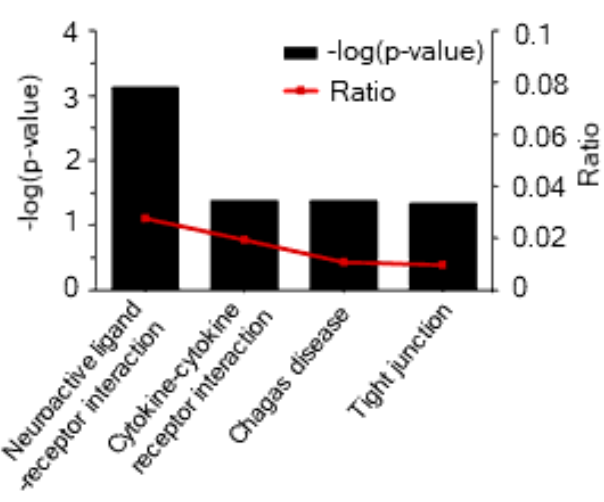

C

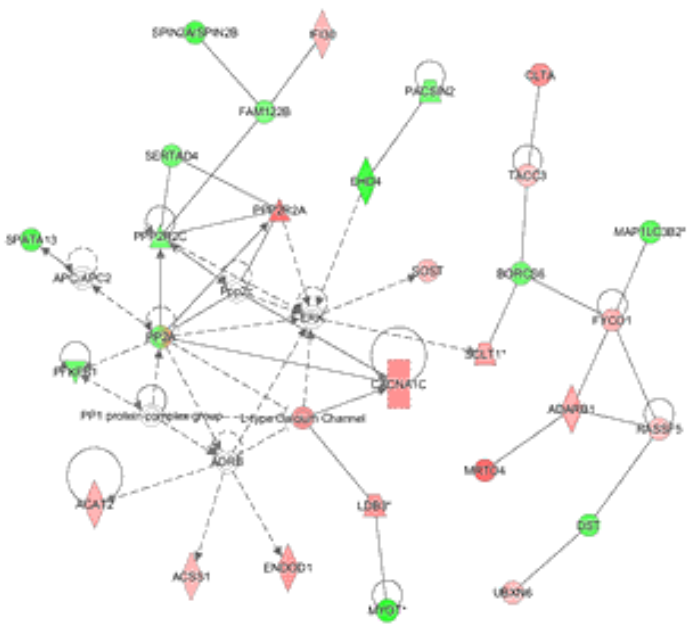

CD59

MMP1

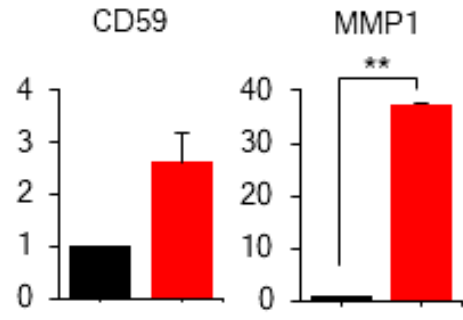

MCF-7 $\square$ MCF-7/TamR

e

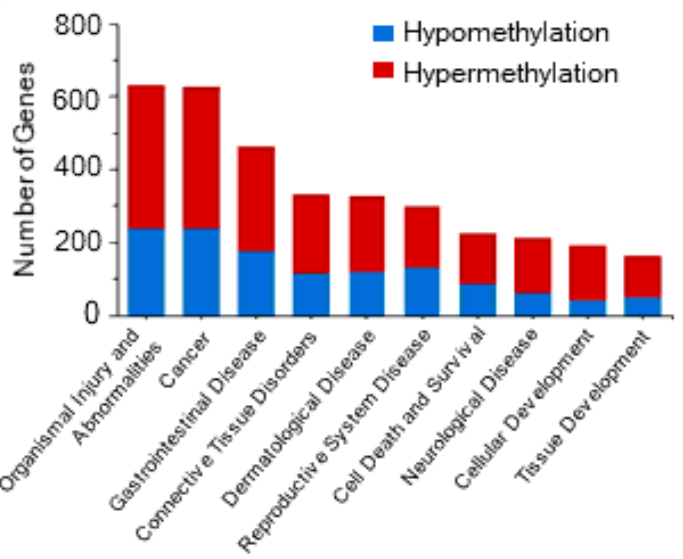

Figure 1

\section{Figure 1}

Highest confidence network and pathway of genes displaying altered methylation in MCF-7/TamR. a Clustering of genes in which methylation is affected in TamR. A heatmap is constructed with 405 CpGcontaining genes showing significant methylation changes $(|\Delta \beta| \geq 0.2 \mathrm{P}<0.05)$ from duplicated microarrays of MCF-7 vs. MCF-7/TamR. b Expression of genes showing highest methylation changes in the microarray assay. Five genes are selected from Table S3 and their expression is examined by qPCR. c 
Genome-wide methylation analysis with 356 genes identifies "Skeletal and Muscular Disorders, Cellular Assembly and Organization, Connective Tissue Development and Function" pathway as the top network. Genes hypermethylated in MCF-7/TamR are shaded in red, whereas those hypomethylated are shaded in green, with the color intensity signifying the magnitude of methylation change. Solid lines represent direct interactions, and dashed lines represent indirect interactions. Top canonical pathways (d) and disease and biofunction (e) for the genes in which methylation is significantly altered in MCF-7/TamR.

a

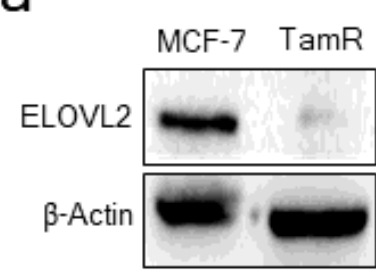

b

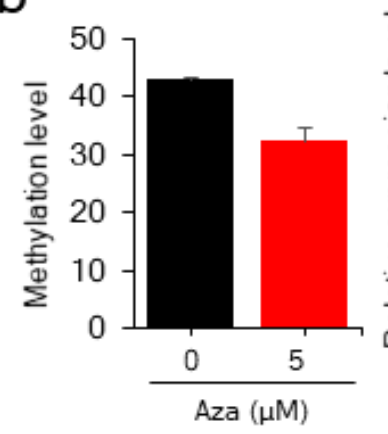

d

Tams
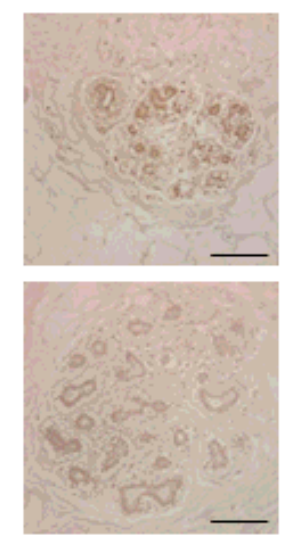
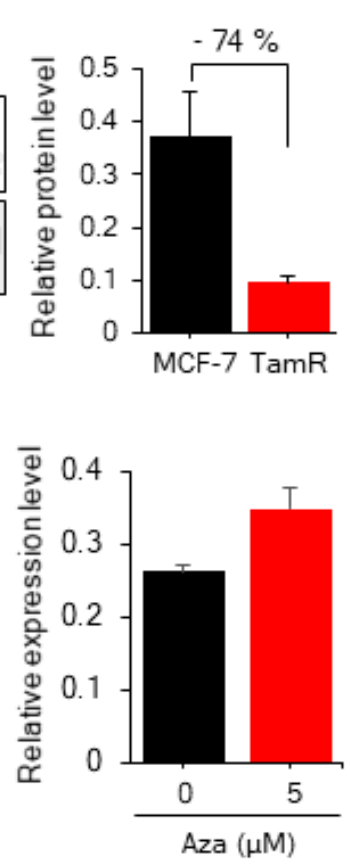

TamR
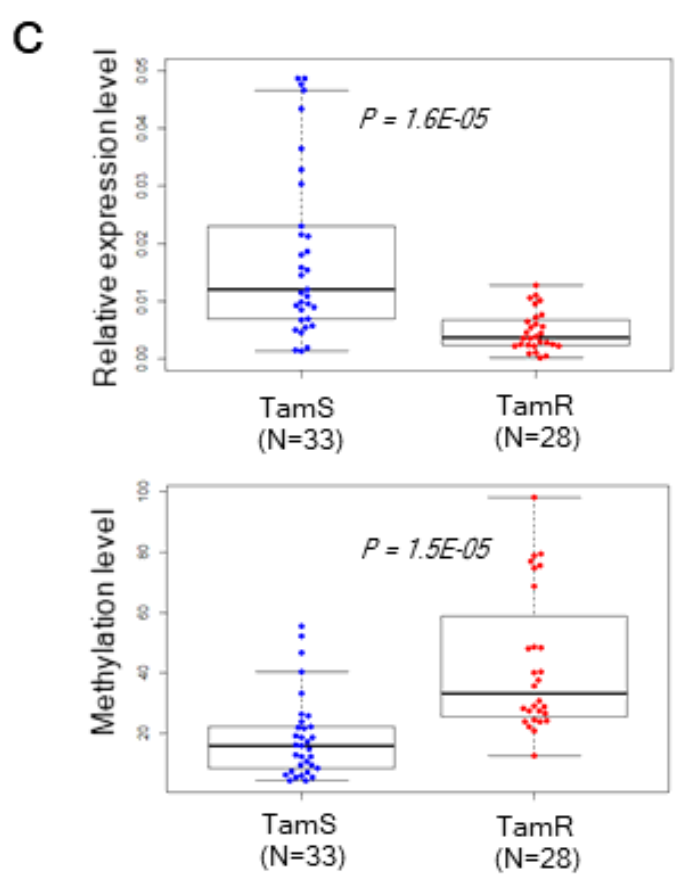

e

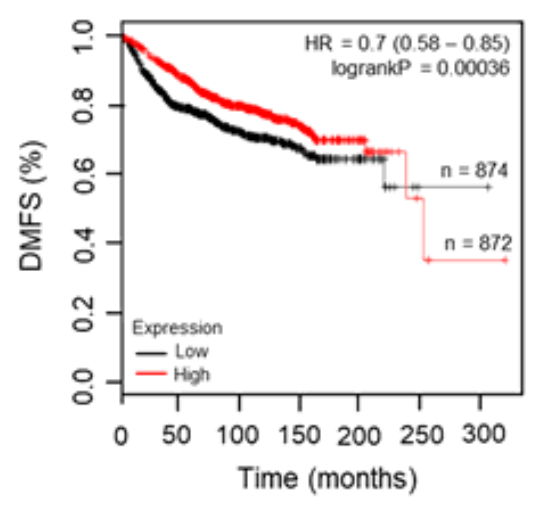

Figure 2

Figure 2 
ELOVL2 is hypermethylated and downregulated in TamR breast cancer. a Downregulation of ELOVL2 in MCF-7/TamR. Expression of ELOVL2 is examined by Western blot analysis. b Demethylation of CpGs is induced by Aza in the MCF-7/TamR cells and ELOVL2 expression is analyzed by qPCR. C Hypermethylation and downregulation of ELOVL2 in breast cancer tissues. MSP and qPCR are performed for breast tissues from Tam-sensitive and Tam-resistant cancer patients. $\mathrm{N}$ : number of samples. $\mathrm{d}$ Immunohistochemical analysis of ELOVL2 in Tam-sensitive and Tam-resistant cancer tissues. Three tissue sets are analyzed and the protein expression is denoted by the bar graph. Images from two tissue sets are represented. Scale bar, $50 \mu \mathrm{m}$. e Kaplan-Meier survival analysis of ELOVL2 expression in breast cancer. Samples $(n=1,746)$ are stratified into two groups based on ELOVL2 expression level. The logrank test is performed in all tumor samples using distant metastasis-free survival (DMFS) as the endpoint. High ELOVL2 expression is significantly associated with higher DMFS in cancer patients $(\mathrm{P}<$ 0.005). 

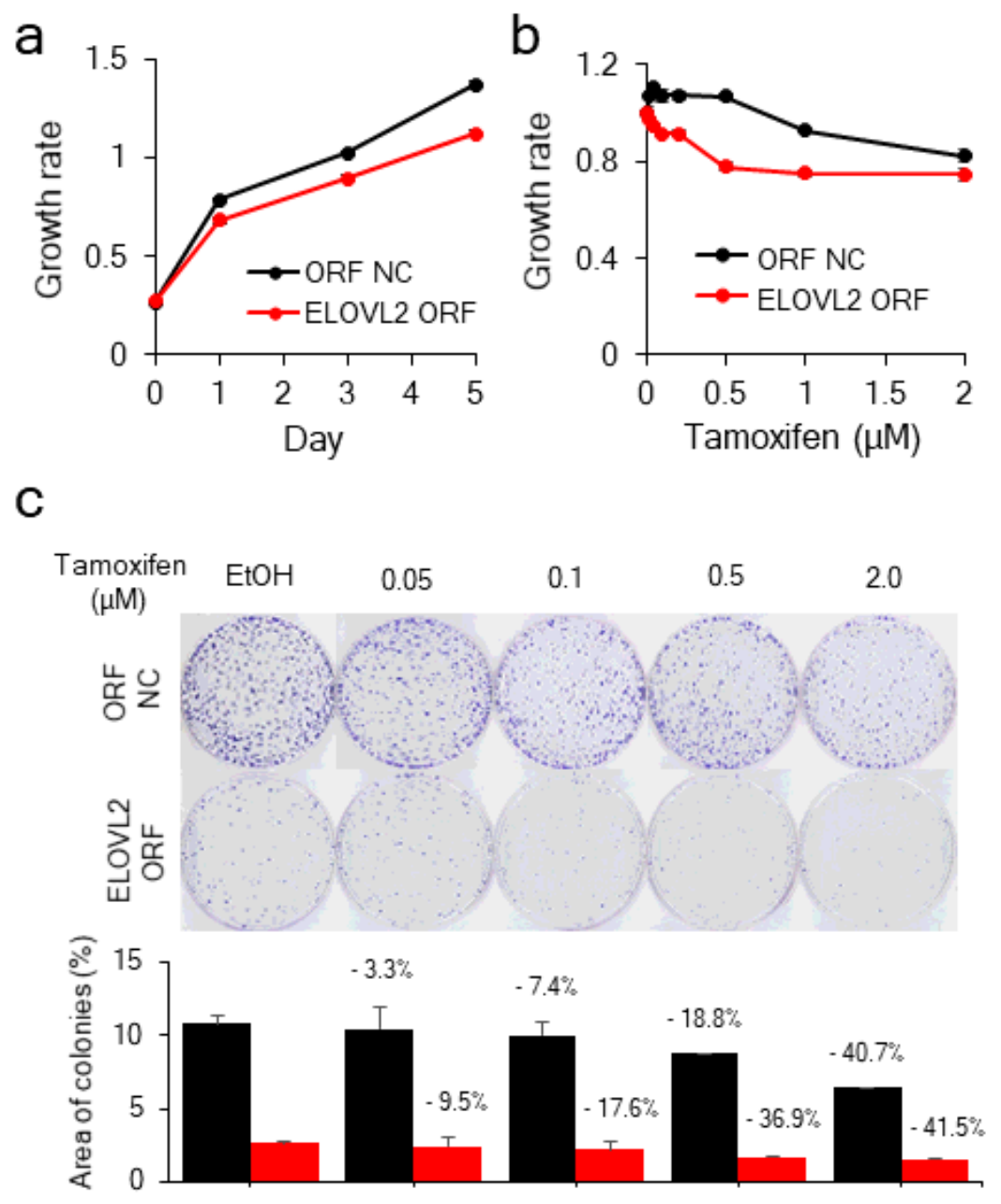

Figure 3

\section{Figure 3}

ELOVL2 inhibits MCF-7/TamR cell growth and sensitizes cells to Tam. ELOVL2 is upregulated in MCF7/TamR by transiently transfecting a recombinant plasmid vector. a Effect of ELOVL2 on cell proliferation is examined by a dye-based CCK assay. b Effect of ELOVL2 on Tam sensitivity is examined after treating the MCF-7/TamR cells with Tam and then measuring the growth rate as described above. $c$ Effect of ELOVL2 on Tam sensitivity is examined by colony formation assay. All the assays are performed in 
triplicates, and the result is depicted as mean \pm SE. Representative images are shown for the colony formation assay. NC, negative control vector. ORF, open reading frame.

a

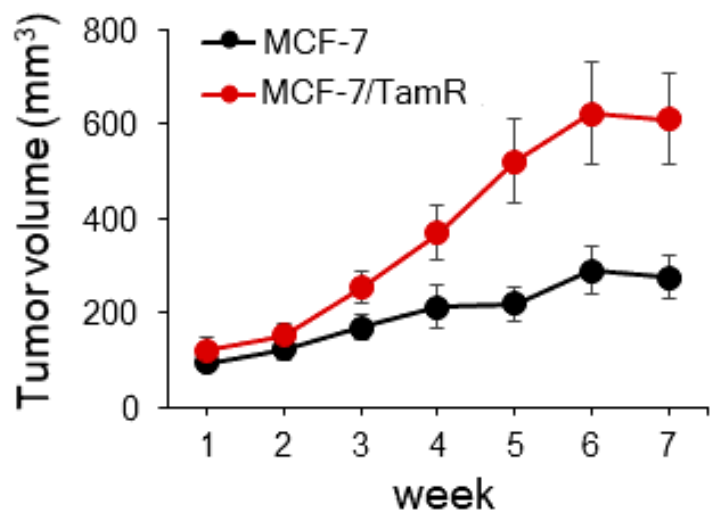

C

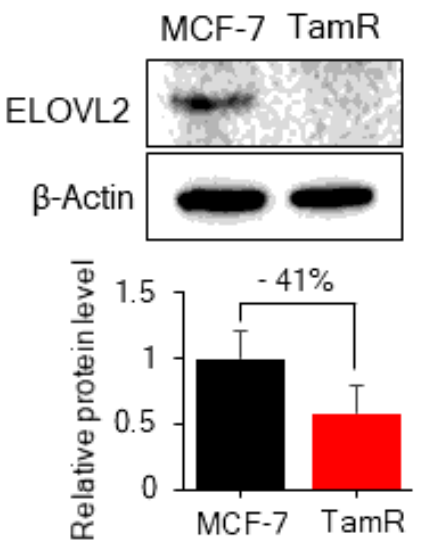

b

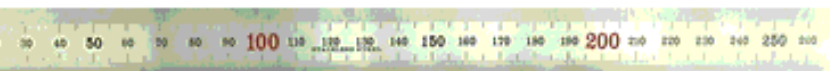

MCF-7

TamR

d

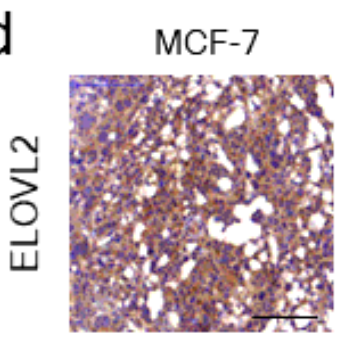

MCF-7/TamR

e
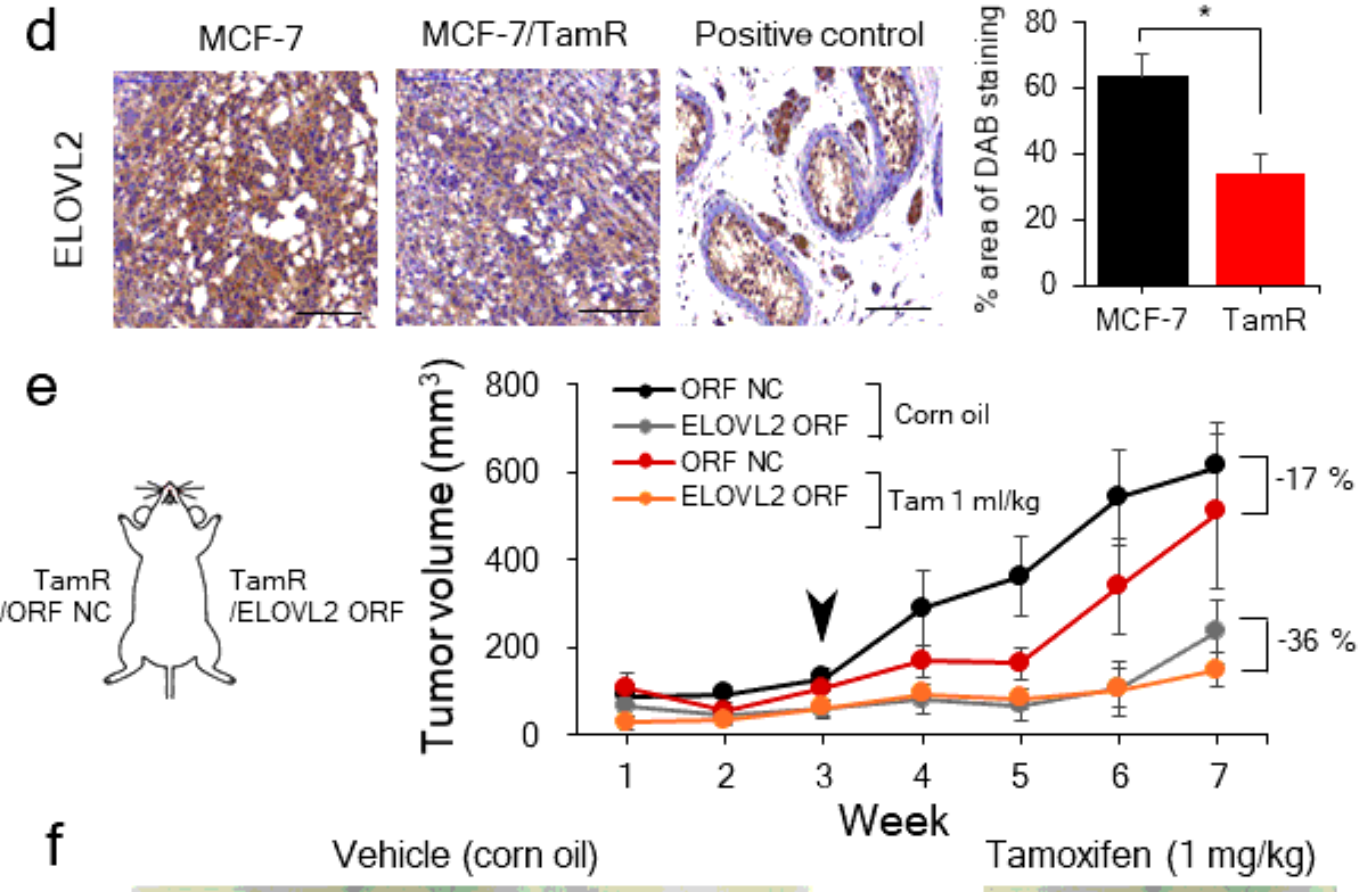

f

Vehicle (corn oil)

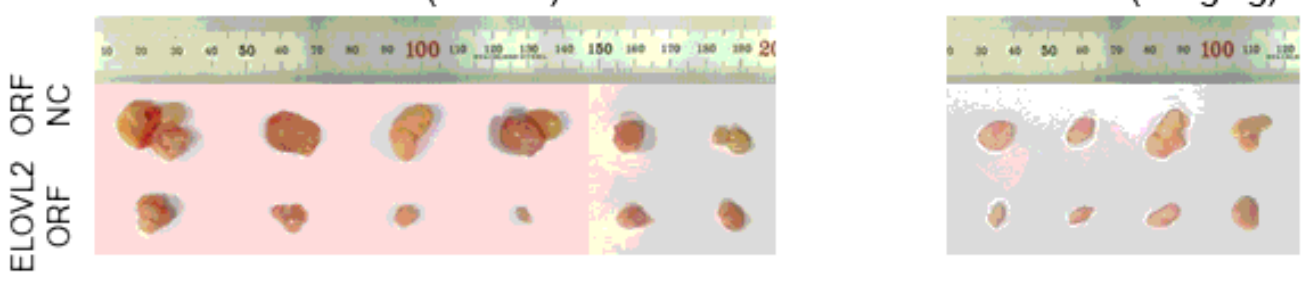

Figure 4

\section{Figure 4}

ELOVL2 suppresses tumor growth and attenuates TamR in an in vivo animal model. a MCF-7/TamR cells grow faster than MCF-7 in a xenograft animal model. MCF-7 and MCF-7/TamR cells are subcutaneously injected into nude mice and the tumor volume is measured for 7 weeks. $n=8$. b Mice are sacrificed 8 
weeks after transplantation to obtain the tumor tissues. Expression of ELOVL2 in the xenografted tumor is examined by Western blot analysis (c) and immunohistochemical analysis (d). Three tumor sets are analyzed and the average protein expression is denoted in a bar graph. Representative images are shown. Scale bar, $50 \mu \mathrm{m}$. e MCF-7/TamR cells that are stably transfected with ELOVL2-expressing cDNA or control DNA are subcutaneously injected into nude mice and Tam is administered 3 weeks after cell injection. The tumor volume is measured for 7 weeks. f At week 8 , mice are sacrificed to obtain the tumor tissues ( $n=6$ for corn oil-treated mice; $n=4$ for Tam-treated mice).

a
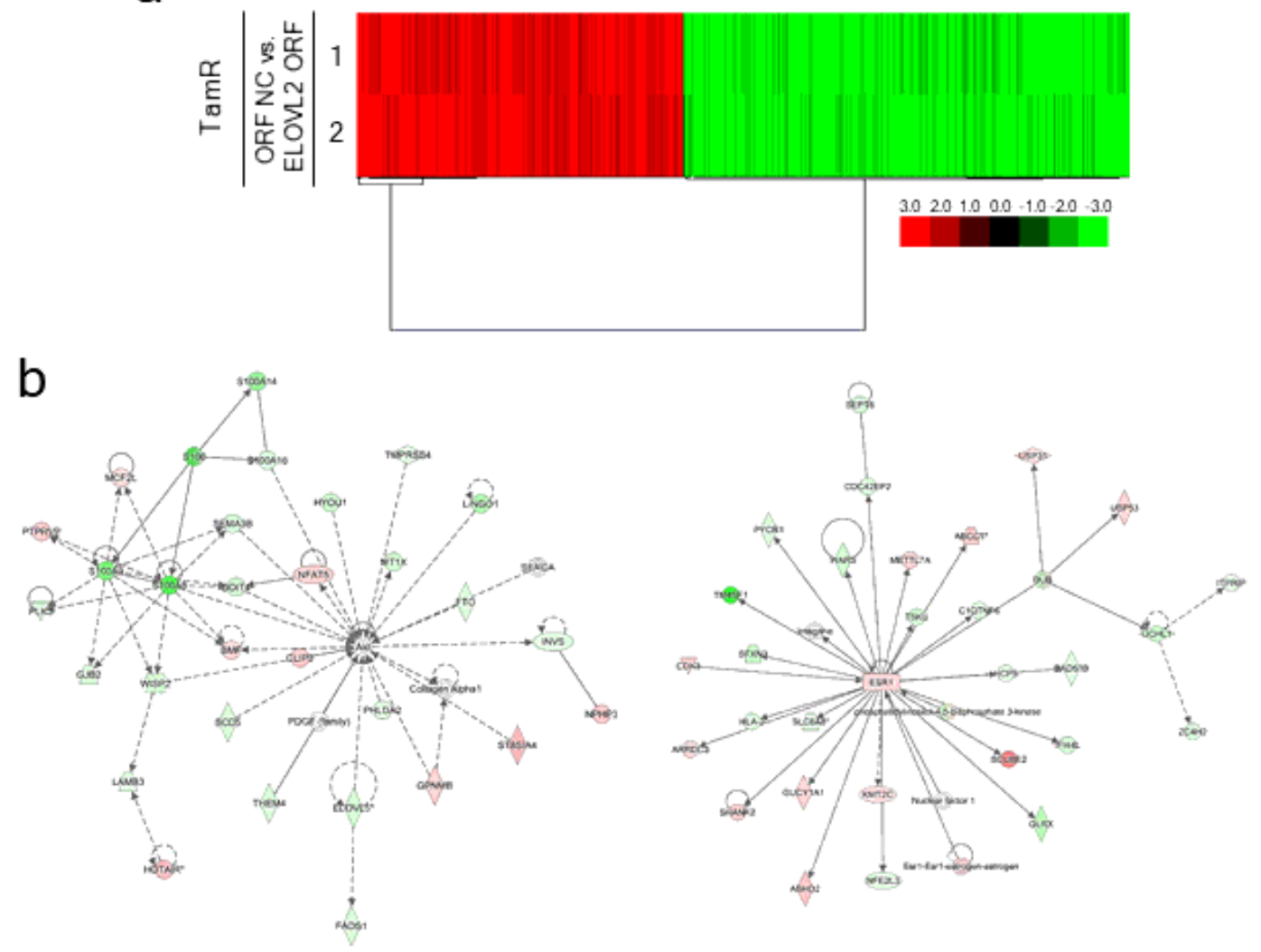

C

d
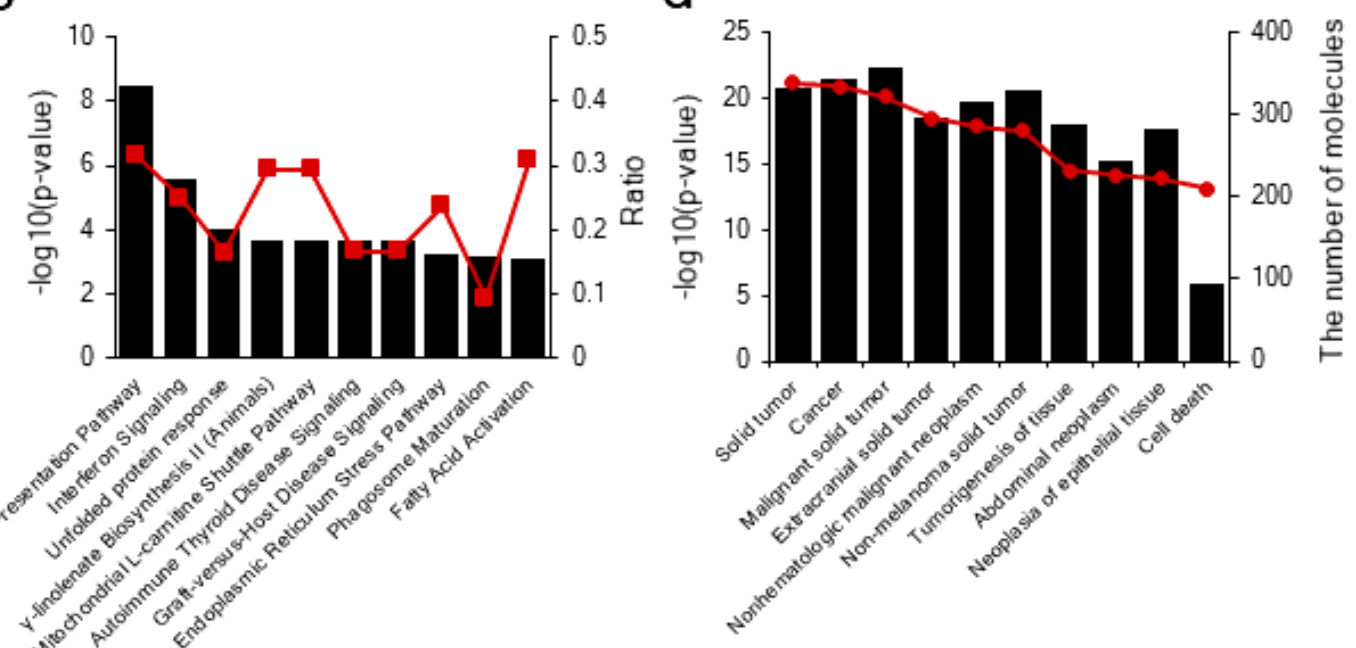

Figure 5 


\section{Figure 5}

Highest confidence network of genes displaying altered ELOVL2 expression in MCF-7/TamR. ELOVL2 is overexpressed in MCF-7/TamR and a genome-wide expression analysis is performed. a Heatmap analysis of 969 genes that are significantly deregulated by ELOVL2. The data are from the microarray in duplicates. b Highest confidence network of genes displaying altered expression identifies "Cardiovascular Disease, Cell-To-Cell Signaling and Interaction, Inflammatory Response" pathway and "Behavior, Reproductive System Development and Function, Cardiac Infarction" pathway as the top networks. Genes that are upregulated are shaded in red, whereas those that are downregulated are shaded in green, with the color intensity signifying the magnitude of expression change. Solid lines representing direct interactions, and dashed lines representing indirect interactions. c Top 10 canonical pathways and d disease and function annotation of the genes of which expression is significantly altered by ELOVL2. The most significant canonical pathway is "Antigen Presentation Pathway" and disease and function annotation is "Solid tumor". 

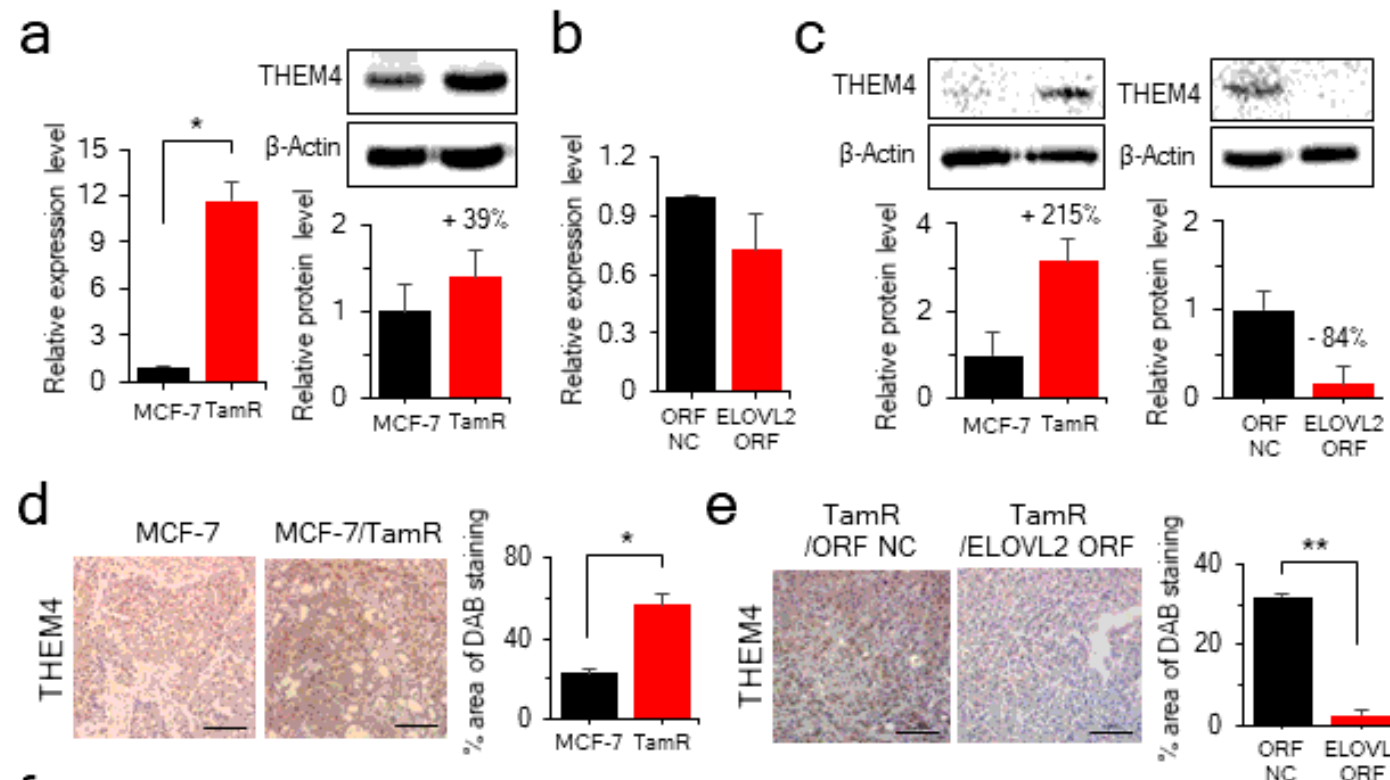

$f$
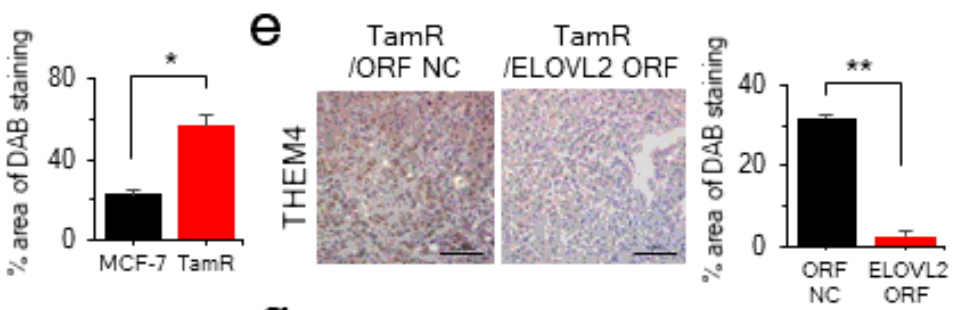

g
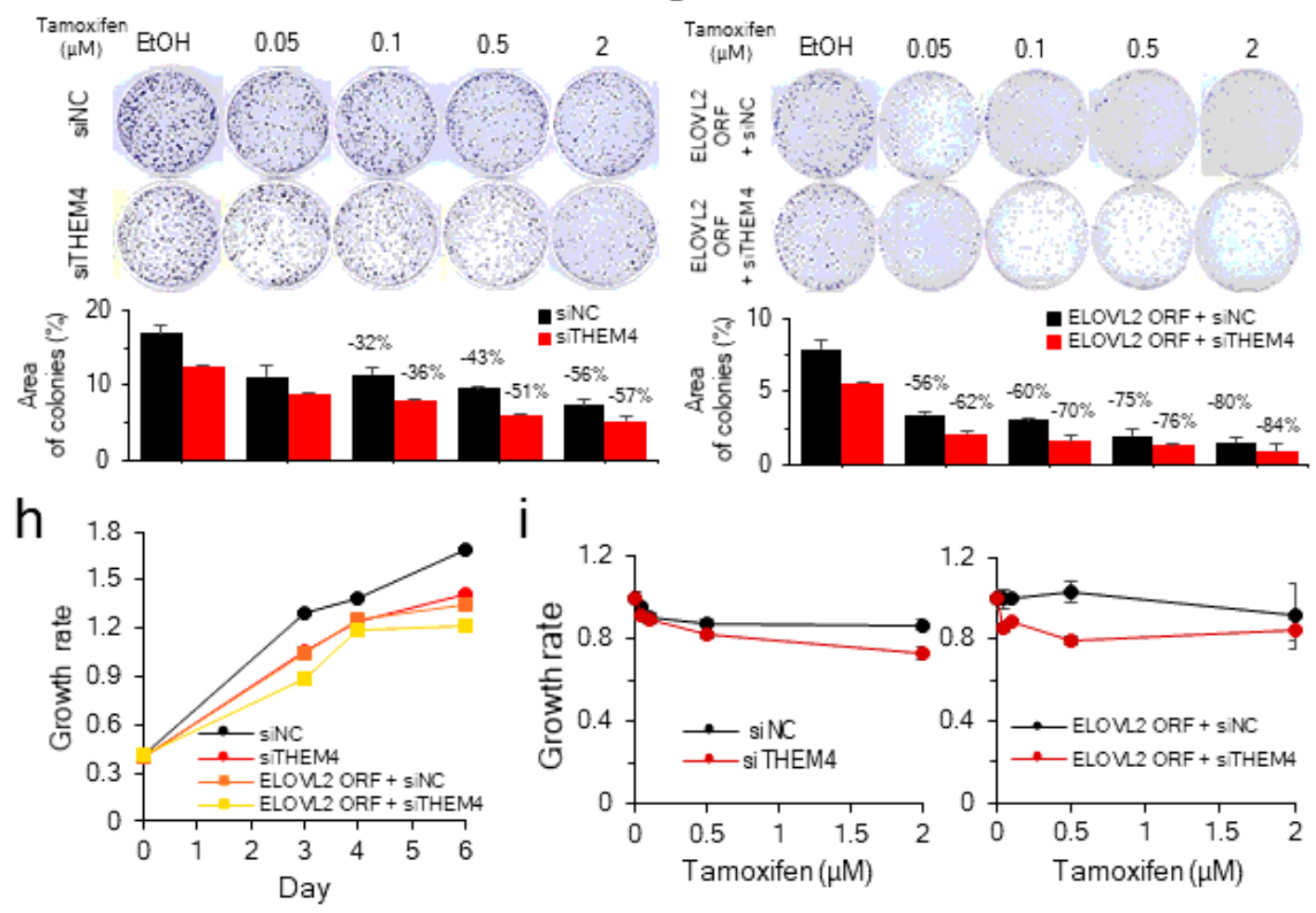

Figure 6

\section{Figure 6}

THEM4 is downregulated by ELOVL2 and increases Tam resistance. a Increased expression of THEM4 in MCF-7/TamR cells. qPCR (left) and Western blot analysis (right) are performed in MCF-7/TamR and MCF7 cells. b Downregulation of THEM4 by ELOVL2 in MCF-7/TamR cells, determined by qPCR. ELOVL2 ORF: cells stably transfected with ELOVL2 cDNA; ORF NC; negative cDNA control. c Increased expression of THEM4 in the xenografted MCF-7/TamR but suppression by ELOVL2. Western blot analysis is performed 
for tumor tissues from ELOVL2 ORF and control. Immunohistochemical analysis of THEM4 in xenografted tumor tissue of MCF-7/TamR (d) and cells stably transfected with ELOVL2 cDNA (e) Scale bar, $50 \mu \mathrm{m}$. Effect of THEM4 on recovery of Tam sensitivity. THEM4 is downregulated via a siRNA in MCF-7/TamR (f) and ELOVL2-overexpressing MCF-7/TamR cells (g). Sensitivity to Tam is examined by colony formation assay. Representative images from three independent assays are shown. $\mathrm{h}$ Effect of THEM4 on cell proliferation is examined by a dye-based CCK assay. i Effect of THEM4 on TamR is examined by exposing the cells to Tam after downregulating the gene with siRNA. All the assays are performed in triplicates, and the result is depicted as mean $\pm \mathrm{SE}$. 


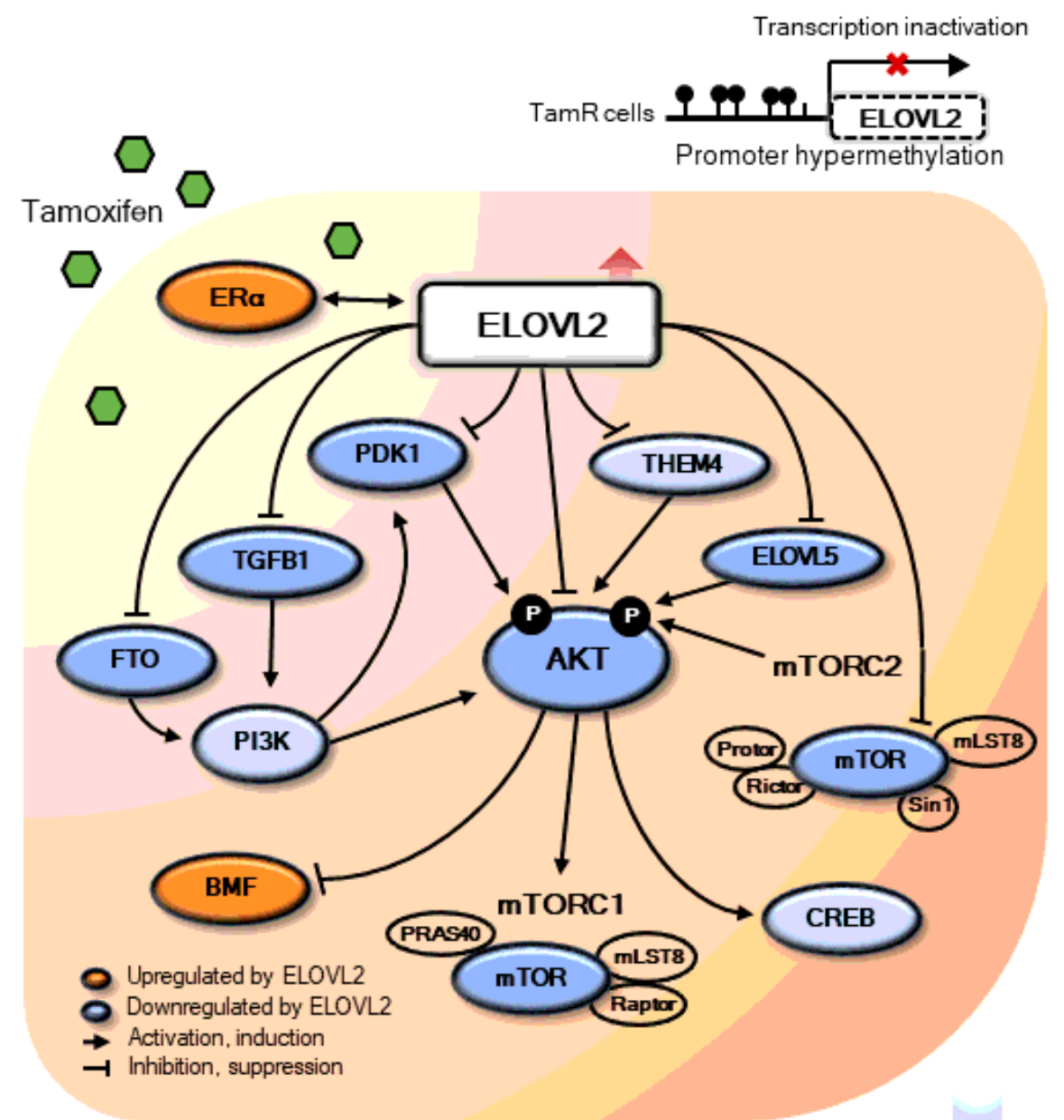

Proliferation and Tam resistance

Figure 7

\section{Figure 7}

Schematic illustration of the regulatory pathway by ELOVL2. The uptake ratio of Tam across plasma membrane in MCF-7/TamR cells is similar to that in the parental MCF-7 cells. ER $\$ and ELOVL2 crosstalk to regulate each other. ELOVL2 blocks the PI3K/AKT/mTOR pathway via inhibiting THEM4 and PI3K. In TamR cancer, ELOVL2 is downregulated by hypermethylation, resulting in loss of inactivation of AKT and 
also downstream genes such as CREB and mTOR, or activation of downstream genes such as BMF, eventually leading to Tam resistance and increased cell proliferation.

\section{Supplementary Files}

This is a list of supplementary files associated with this preprint. Click to download.

- SupplementaryInformationJExpClinCancerResJeongelal.docx

- SupplementaryInformationJExpClinCancerResJeongelal.docx 